taining ideal taxa for biogeographical analysis (unlike the butterflies of D. J. Holloway, which are likely to be of too recent origin to have existed in the peninsula before it became disconnected from Africa). Centres of origin should be traccable, but the discussion never reaches a sufficiently detailed level to convincingly do so. Professor Hilary Crusz's work on the ecology of the extraordinary endemic agamid genera in the hill forests of wet zone Ceylon could, for instance, have provided the basis for such a discussion but his work is not mentioned.

The biogeography of the Indian region is not, of course, that of the peninsula alone; separate chapters are devoted to the Indo-Gangetic plain, and the Himalayan region. But though much information is presented as in the rest of the book, these chapters are marred in the same manner. The chapters on the impact of man, and the ecology of 'tribal' man (by P. Lal) are welcome and useful, as are those on recent faunal impoverishment (A. K. Mukherjee), the ecology of vertebrates of the Indian desert (I. Prakash) and the mammals of Assam (G. V. Kurup).

The book is well designed and printed, though spelling errors, particularly in scientific names, are not infrequent.

P. S. AshToN

\section{Anatomy of experiments}

Design of Experiments: A Realistic Approach. By V. L. Anderson and R. A. McLean. Pp. xvii +418 . (Statistics: Textbooks and Monographs.) (Dekker: New York, February 1974.) $\$ 19.75$.

I HAVE long contemplated the value of a book that would discuss in detail a few experiments in which statistical concepts were vital to the design, the analysis and the interpretation. The declared objective of the book under review is "to express rather complex ideas on how and why scientific investigators should design experiments". Is this the book I want?

Certainly it has many merits. The authors employ a wide range of examples to illustrate the theory and practice of experimental design, choosing these largely (but not entirely) from manufacturing industries of which they obviously have much experience. For the teacher, the examples analysed fully and others outlined as problems for the student will prove useful sources of ideas. Nevertheless, I was disappointed to find attention concentrated on questions that can be answered in terms of currently available theory of linear models for observations. On the structure of analysis of variance and on variance components, the presentation is good. Little is said about reasons for choosing particular designs. The interpretation of results remains on the statistician's desk, and is not carried to the field of application. Computational methods and problems are not mentioned: even the existence of standard programs and the desirable features of computer input and output are neglected. The anatomy of analysis of variance is thoroughly displayed, but the developmental and operational physiology of experimentation remains unexplained.

The book proceeds through standard designs, from completely randomised to lattices and factorials, in a manner at times refreshingly different from other texts. Without entering deeply into combinatorial problems, clear accounts are given of the partitioning of sums of squares. The dependence of analysis on randomisation and of significance tests on variance components is well emphasised, and the emphasis on the inference space is often illuminating. The text is enlivened by many comments that reflect the authors' familiarity with the particular examples, increasing my regret that they did not choose to make that familiarity a distinctive feature at the expense of some of the morc conventional algebra. Had they done so, they might have let the reader see the severe limitations of the Newman-Keuls and other multiple comparison tests which so seldom represent any realistic model of data; they might also have illustrated the full interpretation of analyses of transformed data. The final chapter on response surfaces is an interesting brief survey, tantalising because too short to allow critical comparison of methods and objectives.

D. J. FinNEY

\section{Plant becomes soil}

Biology of Plant Litter Decomposition. Edited by C. H. Dickinson and G. J. F. Pugh. Vol. 1: Pp. xiiv $+1-244+46$. Vol. 2: Pp. $x+245-775+75$. (Academic: London and New York, March 1974.) Vol. 1: £7; Vol. 2: $£ 10.50$.

RECYCIING of elements bound in living matter is essential to the continuation of life in our, at present, virtually closed global ecosystem. Since plants form the bulk of the world's biomass and, at least on land, the bulk of this biomass is recycled by the decomposition of plant litter rather than by consumption by herbivores, there can be no doubt as to the importance of the subject matter of these volumes. The excellent introductory chapter on 'Litter-Interface of Animate/Inanimate Matter' gives a broad and stimulating view of the whole field of decomposition studies. Some later chapters, by the nature of their narrower topics, seem by comparison more mundane in their approach to the ecological issues. Following the introduction, the work is in three parts.

In the first part, plant litter decomposition is treated on the basis of different types of plant litter. Thus there is a chapter on lower plant litter, so important in the transition from rock to soil, followed by chapters on decomposition of herbaceous litter, angiosperm tree leaf litter, coniferous leaf litter, wood and roots. Finally, in part I is a chapter on the nature and decomposition of digested plant litter in the form of animal dung and in the faecal pellets from certain soil fauna. It seems artificial to exclude from consideration in the work the decomposition of plant material which has actively been detached from plants by herbivores or by man for feeding to his herbivorous livestock. Such lines of thought, however, lead to the large, but dubiously separable areas of biodeterioration and ruminant nutrition.

In part 2 , the chapters survey different groups of organisms involved in plant litter decomposition ranging from bacteria, actinomycetes, terrestrial fungi, aquatic fungi, protozoa, nematodes, oligochaetes, microarthropods, macroarthropods and molluscs, to aquatic crustaceans. The emphasis in these chapters varies: some provide a brief introduction to the classification of the group whereas others assume the reader's familiarity with the group and devote attention directly to the ecological significance of the organisms in litter decomposition.

This approach to ecological problems of plant litter decomposition seems less thought provoking for the general reader than is the approach through litter types or the chapters in part 3 where decomposition of plant litter is discussed in relation to the environment in which decomposition takes place. These last chapters cover decomposition both on the surface of soil and in the depth of soil, in fresh water and in marine environments. The two final chapters discuss special environmental problems of the breakdown of agricultural crop debris and urban waste. Peat and coal are economically important deposits of partly decomposed plant debris. Surprisingly, these are largely ignored in the environmental chapters, though a forthcoming review on peat, by one of the contributors, may account for part of this omission.

The editors have performed a considerable service in bringing together and indicating the relevance of some 3.000 references. I suspect that the work will be appreciated as a source book mainly by students, though researchers in this field will glean grains of information.
R. C. CODNer 